\title{
Susceptibility of a stop consonant to adaptation on a speech-nonspeech continuum: Further evidence against feature detectors in speech perception
}

\author{
ROBERT E. REMEZ \\ Indiana University, Bloomington, Indiana 47405
}

\begin{abstract}
The present experiment uses the perceptual adaptation paradigm to establish the validity of a previous test of the feature detector model of speech perception. In the present study, a synthetic stimulus series varied from a CV syllable, [ba], to a nonspeech buzz. When the endpoint tokens were employed alternatively as adaptors, the category boundary was shifted relative to unadapted identification in each adaptor condition. This result suggests that a prior test which used a vowel as the speech endpoint was legitimate because a stop consonant, an exemplary speech sound, was also susceptible to perceptual adaptation in a speech-nonspeech context. Feature detector models predict, incorrectly, that this outcome is impossible. Therefore, this finding may be taken to undermine the interpretation of adaptation as fatigue in a set of detectors tuned to detect the distinctive features of linguistic analysis.
\end{abstract}

One recent approach to speech perception, the phonetic feature detector proposal, derives its design from the hierarchical cascade model of neurophysiology (Eimas \& Corbit, 1973). This conceptualization typically uses a series of increasingly abstract analyses of the speech signal, first to extract a sequence of instantaneous spectra of the stimulus; then to derive a set of time-varying auditory patterns from the preceding simpler level of analysis; and finally to detect in the auditory patterns the phonetic features which, in combination, constitute the segmental sequence of the talker's message. This characterization of speech perception postulates that the highest level of analysis consists of a small set of phonetic analyzers, the inventory of which is described by the universal distinctive feature set from linguistic theory. As such, the proposal suggests that the specializations of the neurological substrate for speech perception are matched to the elements of the sound pattern of language (see Cooper, 1979, and Eimas \& Miller, 1978, for reviews). With the addition of the single assumption that the specialized detectors develop innately, this hypothesis has also been used to explain the precocious speech perception abilities of human newborns (Eimas, 1975; Eimas, Siqueland, Jusczyk, \& Vigorito, 1971). Moreover, the

\footnotetext{
This research was supported by NIMH Grant MH 32848-01 to the author and by NIH Grant NS 12179-04 to David B. Pisoni. I would like to thank Professor Pisoni and Steve Simnick for their assistance during the course of this study; I am also grateful to Dr. C. A. Fowler for her comments on the manuscript. Address correspondence to Robert E. Remez, Department of Psychology, Indiana University, Bloomington, Indiana 47405.
}

binary nature of many phonemic contrasts (Chomsky \& Halle, 1968) is easily incorporated into the model through an opponent-process design in which pairs of feature detectors are yoked in oppositions corresponding to the linguistic analysis. The notion of a genetically endowed set of hypercomplex cells tuned one-to-one to the distinctive features of speech parsimoniously addresses these theoretical and empirical considerations in perception, phonetics, development, and neurophysiology.

The behavioral test of this model has used the technique of perceptual adaptation, which was understood as the induction of fatigue in selected parts of the detector ensemble. The ensuing perceptual changes-category boundary shifts ${ }^{1}$ along acoustic continua of speech sounds-were well predicted by distinctive feature theory in the original experiment. This gave credence to the claim that the particular assortment of neural perceptual analyzers was identical, point for point, to the analytic units described by linguists. Indeed, several subsequent studies appeared to require an abstract level of analysis to explain the adaptation observed (Diehl, 1975; Rudnicky \& Cole, 1977; Ganong, Note 1). The inclusion of a phonetic level in the model therefore appeared to be justified empirically. However, a variety of adaptation effects have failed to find adequate explanation in a phonetic detector account; moreover, the set of distinctive features cannot be used to predict the conditions under which adaptation does (and does not) take place (e.g., Ades, 1974; Bailey, 1975; Cutting, Rosner, \& Foard, 1976; Ganong, 1978; Hall \& Blumstein, 1978; Tartter \& Eimas, 1975). Adaptation in these cases was explained by recourse 
to auditory, rather than phonetic, properties common to the fatiguing stimulus and the continuum used to test the parameters of fatigue. The difficulty in determining the level of perceptual analysis at which adaptation occurs and the frequent failure of the phonetic feature account to predict the conditions under which adaptation takes place have detracted from the otherwise appealing simplicity of the original phonetic conception.

Despite the evidence against a literal neurophysiological interpretation of phonetic features, ${ }^{2}$ recent attempts have been made to preserve the basic feature detector model by reformulating it (Ades, 1977; Cooper, 1979; Eimas \& Miller, 1978; Ganong, 1978). In general, the modifications reflect a compromise between the original model, designed primarily to solve the phonetic invariance problem in speech perception, and a mechanism conceived solely to represent the auditory nature of adaptation effects. For example, the model proposed by Eimas and Miller (1978) employs multiply tuned auditory detectors; each is sensitive to a variety of auditory patterns, including those directly relevant to determining phonetic features and, also, those patterns relevant only to detecting the phonetic context in which the feature occurs - that is, the "parameters of the speech signal which specify vowel environment, syllabic position, fundamental frequency, intensity, voicing, manner of articulation, and perhaps other dimensions" (p. 317). Then clusters of multiply tuned auditory detectors converge upon individual higher level mechanisms, each of which yields a single context-independent phonetic feature value. The converging-auditory-detector design enables the model to account for the findings that fatigue is primarily "low-level"; also, the model is apparently a natural one, assigning perceptual categories by virtue of detectors whose tunings and mappings to higher levels have been established by specific inheritance and linguistic experience.

Do these changes in the original conception produce a detector model worth retaining? Though the new model is better able to account for contingent and selective adaptation effects, the adaptation technique itself has recently been reevaluated (Diehl, Elman, \& McCusker, 1978; Simon \& Studdert-Kennedy, 1978); it seems that changes in identification brought about by the repetition of a speech sound may not result from detector fatigue. The simple effect of contrast between adaptor and test token appears to be indistinguishable from the effect of adaptation, under several different test conditions, and both for consonants (Diehl et al., 1978; Diehl, Lang, \& Parker, in press; Simon \& Studdert-Kennedy, 1978) and vowels (Sawusch \& Nusbaum, 1979). If the dimensions of phonetic adaptation are found to collapse onto the dimensions of stimulus contrast, then the "features" would appear to be without limit, and there would be little basis for the specific claim that complex, though fatiguable, feature detectors mediate the perceptual constancies observed in speech perception; neither would there be grounds for supposing that these two techniques, adaptation and induced contrast, are of singular value for exposing the underlying principles of perceptual constancy in speech.

In the spirit of the reservations about the feature detector approach, an empirical test was recently performed (Remez, 1979) which demonstrated adaptation of a category boundary between a speech sound (the vowel [æ]) and a nonspeech sound (a buzz). In this experiment, the [æ] and buzz continuum endpoints were used alternatively as adaptors in the adaptation paradigm. The boundary shifts noted for each adaptor on this speech-nonspeech continuum were comparable to those observed on continua of speech sounds only, but were not explicable by resort to a feature detector explanation of any kind. The finding could be assimilated by a phonetic detector model (1) only if its feature detectors include a $[+$ speech $],[-$ speech $]$ distinction, or (2) only if the speech detectors are individually connected opponent-style to a corresponding set of nonspeech detectors. It was argued that neither of the alternatives afforded a plausible extension of the detector inventory. The first alternative, which would essentially claim that a new feature was discovered, was eliminated on linguistic grounds; no language makes phonemic use of a distinction between abstract segments that are speech and those that are not. The phonemic use of a vocal sound necessarily confers [ + speech] status to that sound, despite any similarity the sound might have to nonspeech whistles, clicks, hisses, or buzzes. The second alternative, that the phonetic detector set is merely a component of a larger detector ensemble responsive to speech and nonspeech sounds alike, was eliminated on neuropsychological grounds (reviewed by Hecaen \& Albert, 1978); the higher cortical mediation of speech appears to be anatomically segregated from the nonspeech auditory counterpart.

An auditory-detector model of the type proposed by Eimas and Miller (1978), comprising auditorypattern detectors fed into higher level linguisticdecision mechanisms, fares no better in accounting for the data demonstrating speech-nonspeech adaptation. This is because the model employs the rationale of phonetic distinctive features in designating selective tunings and the mapping of lower-level analyzers onto the higher level mechanisms. In other words, the model takes the speech processor to be a device whose structure is fixed by inheritance and early linguistic experience; it is a neural, i.e., fatiguable, replica of distinctive feature theory, and its selective sensitivities are said to derive directly from the auditory circumstances in which those features 
actually occur. We might infer, then, that the auditory detectors are selectively tuned only to those stimulus patterns which affect phonetic feature assignment, and not, for example, to every stimulus that excites the auditory system. As such, the model is prohibited from explaining adaptation of the speech-nonspeech boundary through resort to what is otherwise its chief virtue-the capability of proliferating new auditory "tunings" ad infinitum to match the context-contingencies observed in ongoing selective adaptation research. Because there is no known natural case in which phonetic feature assignment depends directly or contextually on nonspeech signals, the model must fail to admit a tuning to the nonspeech end of the [æ]-buzz continuum; there would be no phonetic justification for including it. The auditory feature detector model simply predicts that adaptation of the speech-nonspeech continuum should not occur. This prediction is independent of the claim, be it true or false, that the effect is caused by simple auditory fatigue (see Remez, 1979). In the absence of an auditory principle for establishing which patterns are relevant to phonetic recognition and which are not, we should reject, on the basis of common sense, the notion that an auditory detector has been tuned by inheritance and linguistic experience to detect broad-band buzz spectra in order to promote phonetic feature assignment.

The principal argument of the present report is that the speech-nonspeech test of the earlier study, though it may have been suggestive, may not have posed a substantial challenge to the feature detector model. This is because the speech sound used in the test continuum was a vowel, and vowels appear to be the least speech-like of speech sounds (Liberman, 1970). Unlike the categorical consonants, vowels are perceived more continuously (Fry, Abramson, Eimas, \& Liberman, 1962); vowels do not exhibit a rightear superiority when presented dichotically, though consonants do (Studdert-Kennedy \& Shankweiler, 1970); memory processes for consonants and vowels differ (Crowder, 1971; Pisoni, 1975), as do their perceived psychophysical scales (Vinegrad, 1972; Perey \& Pisoni, Note 2). Vowels and consonants also appear to require different types of motor acts (Fowler, Rubin, Remez, \& Turvey, in press; Ohman, 1966, 1967; Perkell, 1969). In short, the precondition of the earlier test of Remez (1979), that one of the continuum endpoints be speech and the other not, may not have been met. On the evidence just cited, we might presume vowel feature detectors to be closely related to nonspeech processes. If so, then adaptation along the [æ]-buzz continuum may actually have been mediated by a generalized nonspeech processor which happened to include feature detectors for vowels. The speech-nonspeech adaptation test employing a vowel as the speech sound would lose its force if that argument was credible, and the feature detector model of speech perception would remain secure, at least with respect to the arguments and evidence presented earlier by Remez (1979).

To insure that the test series ranges from a proper speech sound to a nonspeech sound, the present experiment uses a continuum in which the speech sound is a CV syllable, [ba], a synthetic token with formants of narrow bandwidth. The nonspeech sound here is a buzz, a synthetic sound which has formants of broad bandwidth. The prediction of the feature detector model is that fatigue in the analytic "cascade" caused by either adaptor should not affect the identification of the continuum, because there is no mechanism by which the fatigued speech detectors are integrated with or compared to the nonspeech detectors. In other words, adaptation should not occur. This prediction would be the same even if the actual mechanism of adaptation is sensory, as many authors have claimed (see above). Because the range of alternatives for the speech processor, whether subserved by auditory or phonetic detectors, is presumed to be fixed by inheritance and the early linguistic experience of the listener, it should not mediate any auditory patterns irrelevant to phonetic feature assignment; nor should it be free to undergo demand-specific alteration occasioned by particular tasks.

In summary, the present experiment extends the prior finding of Remez (1979) by using a speechnonspeech test series in which the speech endpoint is a CV syllable. Because stop consonants are exemplary speech sounds, we can be reasonably certain that if detectors mediate speech perception, this stimulus series will receive detector mediation-or, at least the [ba] portion of the test series will. Unlike our earlier experiment, which employed a vowel, this consonantal version would seem to be an appropriate test of the hypothesis that the speech signal is processed by an ensemble of detectors tuned to extract the distinctive features of linguistic analysis.

\section{METHOD}

\section{Subjects}

Eight Indiana University undergraduate students served as listeners in the experiment. They had been recruited by handbill advertising, and were paid for their participation. All were native speakers of English, and none had a history of impaired speech or hearing.

\section{Stimuli}

A software version of the Klatt digital synthesizer was used in cascade mode to create the stimuli. This program runs on a PDP-11 computer in the Speech Perception Laboratory of the Psychology Department, Indiana University (see Kewley-Port, Note 3). The acoustic continuum of 10 tokens varied from the $\mathrm{CV}$ syllable [ba] to a nonspeech buzz. All synthesis parameters, with the exception of formant bandwidth, were identical in each of the tokens. The rising formant transition pattern characteristic of $/ b /$ in syllable-initial position was made by starting F1 at $400 \mathrm{~Hz}, F 2$ at $1,000 \mathrm{~Hz}$, and $F 3$ at $2,400 \mathrm{~Hz}$, and linearly 
SPECTRUM AT ONSET (INITIAL 20 MSEC)
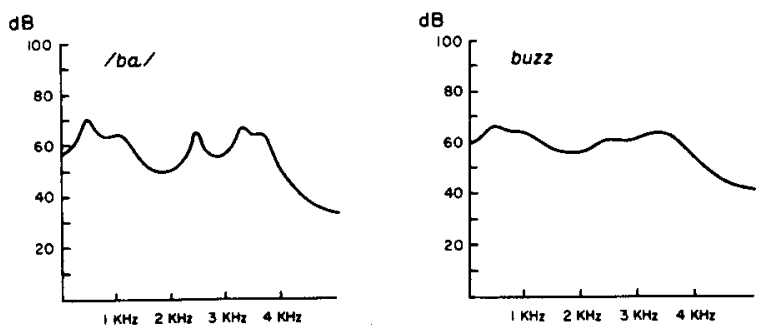

Figure 1. Spectra of the continuum endpoints at onset: [ba] (left panel) and buzz (right panel).

increasing the value of each over $30 \mathrm{msec}$ to $700,1,200$, and $2,600 \mathrm{~Hz}$, respectively. The values of $F 4,3,300 \mathrm{~Hz}$, and $F 5$, $3,700 \mathrm{~Hz}$, were constant for the duration of each token, $290 \mathrm{msec}$. These synthesis values for formant frequency were based on a natural utterance of [ba]. Fo was flat at $110 \mathrm{~Hz}$ throughout each token. Initially, the formant bandwidths were set at $200 \mathrm{~Hz}$, which produced the [ba] endpoint. Iterative $50-\mathrm{Hz}$ increments in each of the five bandwidth parameters, to $650 \mathrm{~Hz}$, were made to create the 10-item test continuum. Each token was synthesized at a sample rate of $10 \mathrm{kHz}$, stored digitally on magnetic disk, and retrieved for presentation during the experimental sessions. Stimuli were output at that time by digital-to-analog conversion with a $5-\mathrm{kHz}$ low-pass filter in effect. Smoothed linear prediction spectra integrated over the initial $20 \mathrm{msec}$ of each endpoint are displayed in Figure 1.

The listeners sat at carrels in a sound-attenuating room. Stimuli were presented over TDH-39 earphones at $80 \mathrm{~dB}$ SPL, and responses were scored on two-button response modules interfaced to the computer. The buttons were labeled "BA" and "BUZZ."

\section{Procedure}

Each listener participated in two sessions, which corresponded to the use of each endpoint as an adaptor. Testing was done in two groups of four subjects; the first group received the [ba] adaptor in the first session and the buzz adaptor in the second, while the second group received the adaptor conditions in the reverse order. Each testing session included three segments: a practice sequence, an unadapted identification test, and an adapted identification test.

An experimental session began with an introductory practice sequence of 10 trials, five each of the two endpoint tokens in random order. This segment of the procedure acquainted the listeners with the best example of each category. Identifications were scored as "BA" or "BUZZ" on each trial, and listeners were informed of the "correct" response by the onset of a light above the appropriate response button. At the conclusion of this segment, every listener admitted that the distinction between [ba] and buzz was clearly perceptible. Then the unadapted identification test began.

In the unadapted baseline identification segment of the test, listeners judged the continuum members presented in a random order, 15 repetitions of each of the 10 , with $3 \mathrm{sec}$ of silence between trials. A different random order was used in each of the four occurrences of the identification test (two groups of subjects, two sessions per group). At the conclusion of this segment, the subjects were instructed in the routine of the adaptation test. When the subjects said that they understood the instructions, the adaptation test began.

The adaptation test contained, first, a block of 50 repetitions of the adaptor token, either the [ba] or the buzz endpoint as the case may have been, with $1 \mathrm{sec}$ of silence between successive presentations. This repetitive stimulation was designed to induce adaptation, hence no response was called for during adaptor blocks. Immediately after the 50th adaptor presentation, a "ready" light on the response module went on, and 2 sec later the identification series started. A sequence of 10 randomly ordered continuum tokens was then presented with $3 \mathrm{sec}$ of silence between trials. The subjects responded by depressing the appropriate button on the response module. The "ready" light was offset after the 10th item, at which point another block of 50 adaptor repetitions was presented. The alternation of 50 adaptors and 10 identifications continued for the duration of the test. Ten-trial sequences within identification blocks were made by randomly ordering a series of 150 trials (10 continuum items by 15 repetitions of each) and dividing it into successive groups of 10 trials. A different random order was used in each of the four occurrences of the adaptation test.

\section{RESULTS AND DISCUSSION}

A least-squares normal ogive (Woodworth, 1938) was fitted to the data of each of the four identification tests contributed by each subject. The mean of each ogive could then be used as an estimate of the category boundary. A one-way analysis of variance of the ogive means was used to calculate the error term for planned orthogonal comparisons using the $t$ statistic (Hayes, 1973). Both adaptors produced a significant change in the labeling function in the direction of change predicted by the adaptation rationale. Adaptation with [ba] caused the category boundary to move from a mean baseline value of $372 \mathrm{~Hz}$ to a mean adapted value of $282 \mathrm{~Hz}$, whereas buzz adaptation caused the boundary to move from 398 to $470 \mathrm{~Hz}$ ( $p<.01$ in both cases). The group identification functions, drawn for each adaptor condition, appear in Figure 2. Each point on the curve is the average of 120 trials (15 repetitions of each token for eight subjects).

The adaptation of the boundary between [ba] and buzz is neither predicted by nor compatible with the feature detector models of speech perception. In the phonetic detector version, speech sounds are perceived independently of other ongoing stimulation by a discrete special-purpose device tuned to phonetic features. To explain the present set of results, the model would have to incorporate an additional specialization allowing the fatigue of [b] detectors to affect nonspeech sounds and speech sounds alike. Not only would this require the device to sacrifice the linguistic rationale for the speech processor, but it would also contradict evidence from neuropsychology on the anatomical separation of these two kinds of higher level processes of auditory perception. To take a single neuropsychological example, Hecaen and Albert (1978) review the relative importance of left hemisphere structures in supporting language functions; but, for the recognition and identification of objects by ear, a complementary nonspeech ability, they write that particular areas of the right hemisphere appear to be critical. The inference to be drawn here 
BANDWIDTH IN HZ

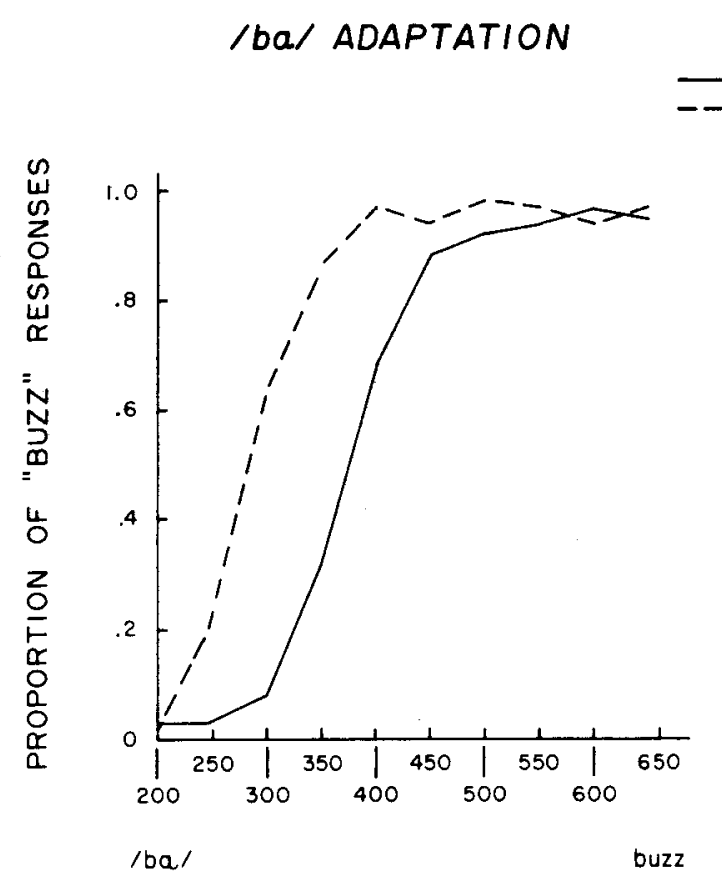

Figure 2. Baseline and adapted identifications in two adaptor conditions: [ba] adaptor (left panel) and buzz adaptor (right panel). is that linguistic and nonlinguistic perception may each be supported by distinct cortical areas which are widely separated from one another. Though it would not be absolutely inconceivable that a finegrained opponent-process network spans speech and nonspeech cortical areas, our present neuropsychological understanding would not encourage the conceptualization of a general perceptual structure serving speech as well as nonspeech sounds. We might therefore take the speech-nonspeech adaptation reported here as evidence of a functional bridge between the two domains, rather than as a point of support for a general auditory perceptual structure in which phonetic feature detectors interact with higher level property detectors for nonspeech sounds.

We might alternatively suppose, as have many authors (Ades, 1977; Eimas \& Miller, 1978; Ganong, 1978; Sawusch, 1977) that a large part of adaptation effects may simply be due to auditory fatigue, though invoking this assumption to explain the present set of results will not also permit us to retain the detector model. It would still be necessary to explain why the perception of buzzes should ever by contingent on the state of the auditory detectors for [ba], and vice versa, if the multiple tuning arrangements and mappings assumed by the auditory version of the model are thought both to be fixed and to be established by early linguistic experience. For, although fatigue could be auditory in this instance, in order for it to affect the [ba]-buzz judgment, the model would have to include a level of integration at which this speech-nonspeech distinction could be assigned. There would be no other way, within the detector framework, for a sound to seem more "[ba]-like" by virture of becoming less "buzz-like." It seems, then, that these findings considerably undermine the claim that adaptation reveals the existence of fixed, phonetically motivated detectors arrayed in opponent-process fashion; and, also, it questions the notion that feature detectors mediate speech perception.

One reason that the detector model proves critically testable by so simple an experiment is that the sources of direct behavioral evidence in support of it are limited to those tests which have employed the selective adaptation technique. The rationale for the adaptation test is that fatigue in portions of an analytic sequence cause the perceived quality of the test stimuli to be altered; the specific alterations are thought to result from the operation of the detector ensemble without the full participation of its fatigued members. Thus, the details of perceptual alterations occasioned by selective adaptation reveal the individual sensitivities of formerly robust analyzers. A test of this kind of "pandemonium" scheme in speech perception looks to see whether the perceptual system will break down along the dimensions established by linguistic analysis; we have seen that the evidence is equivocal and does not verify the original conception (Eimas \& Corbit, 1973). Nevertheless, 
many other studies also seem to warrant the psychological use of distinctive features appropriated from linguistics (e.g., Miller \& Nicely, 1955; Singh, 1966; Studdert-Kennedy \& Shankweiler, 1970; Wickelgren, 1966), but it should be emphasized that the use of techniques other than adaptation prevents those results from supporting the hypothesis of distinctive feature detectors. The serviceability of distinctive feature descriptions of perceptual or memory experiments does not in itself count in favor of a specific proposal of neural mechanism; any physiological model of speech perception, including those which may avoid a literal interpretation of psychoneural identity, must be able to derive these phenomena.

In addition to challenging the detector model, the present study also makes use of a property of speech perception which has begun to receive scrutiny only recently (Aslin, Pisoni, Hennessy, \& Perey, 1979), namely, perceptual plasticity. Although it would be obviously false to allege that every perceptual constancy is in a perpetual state of change, it would seem equally false to represent categories as fixed entities, resistant to differentiation. The present experiment shows that no more than 10 trials are required to enable listeners to begin to differentiate novel waveforms as speech or nonspeech sounds. The adaptation test at the very least suggests that in the naming of the continuum items as speech or nonspeech, the categories are treated as contingent; items which became less [ba]-like also became more buzz-like, and vice versa. Could this have resulted from the increased salience of those particular physical attributes of the waveforms which in this case distinguish the categories (for instance, multipeaked spectrum vs. flat spectrum)? Such a proposal would be compatible with a "channels-of-analysis" view (Kay \& Matthews, 1972; Simon \& Studdert-Kennedy, 1978 ), although this notion is taken here to mean that the listener implicitly determines which functional combination of channels is competent to perform the contingency task. This approach is required primarily because the assortment of channels which may have supported categorization here were unlikely to have been developed beforehand for detecting the difference between speech sounds and nonspeech sounds; the spectral differences between the categories of the continuum do not distinguish them as speech and nonspeech, but merely distinguish them as acoustic patterns which can be categorized easily. It might be argued, to the contrary, that listeners know tacitly what kind of spectrum speech typically has. However, the definitive characteristic of speech as an acoustic stream resists such static description. The speech stream may exhibit "good continuation" because its abstract properties persist despite rapid spectral change from moment to moment: from high-amplitude energy to low; from periodic excitation to aperiodic; from diffuse spectrum to compact. Thus, distinguish- ing speech from nonspeech may not require a perceiver to choose a characteristic kind of static spectrum to be the standard for each. Rather, our technical description of a perceiver's abilities must ultimately confront the apparent continuity of the speech stream in face of the continual acoustic change which, in turn, originates in the continually changing configuration of the articulators as speech is uttered. Though the subjects in the present experiment may have used a static spectral measure as the basis for their perceptions, it is not to be supposed that this criterion would suffice generally for distinguishing speech sounds from nonspeech sounds, and therefore may have been adopted specially for the task imposed by the experiment.

In conclusion, the finding of susceptibility to adaptation of a stop consonant on a speech-nonspeech continuum offers additional counterevidence to the feature detector model of speech perception. Although the status of vowels as speech may be in dispute, thus mitigating the force of some of our earlier arguments and evidence, stop consonants are acclaimed to be unequivocal speech sounds. Were an ensemble of speech feature detectors operating, the present test would have tapped them. Because the detector model incorrectly predicts that adaptation along the speech-nonspeech continuum is impossible, due to the presumed independence of the speech detectors from other perceptual processes, the present findings may be taken to undermine the conceptualization of phonetic adaptation as neural fatigue; we may no longer want to consider the process of speech perception a kind of analysis performed by a genetically endowed set of hypercomplex cells tuned to detect the distinctive features of linguistic analysis.

\section{REFERENCE NOTES}

1. Ganong, W. F., III. An experiment on "phonetic adaptation." Progress Report, Research Laboratory of Electronics, Massachusetts Institute of Technology, 1975, 116, 206-210.

2. Perey, A. J., \& Pisoni, D. B. Dual-processing vs. responselimitation accounts of categorical perception. A reply to Macmillan, Kaplan and Creelman. Paper presented at the 94th Meeting of the Acoustical Society of America, Miami Beach, Florida, December $15,1977$.

3. Kewley-Port, D. KLTEXC: Executive program to implement the KLATT software speech synthesizer. Research on Speech Perception, Progress Report No. 4, Department of Psychology, Indiana University, Bloomington, Indiana, 1978.

\section{REFERENCES}

Ades, A. E. How phonetic is selective adaptation? Experiments on syllable position and vowel environment. Perception \& Psychophysics, 1974, 16, 61-66.

AdEs, A. E. Source assignment and feature extraction in speech. Journal of Experimental Psychology: Human Perception and Performance, 1977, 3, 673-685.

Aslin, R. N., Pisoni, D. B., Hennessy, B. L., \& Perey, A. J. Identification and discrimination of a new linguistic con- 
trast. In J. J. Wolf \& D. H. Klatt (Eds.), Speech communication papers. New York: Acoustical Society of America, 1979.

Bailey, P. J. Perceptual adaptation in speech. Unpublished dissertation, Cambridge University, 1975.

Сномsкy, N., \& Halle, M. Sound pattern of English. New York: Harper \& Row, 1968.

CCOPER, W. E. Speech perception and production. Norwood, N.J: Ablex, 1979.

Crowder, R. G. The sound of vowels and consonants in immediate memory. Journal of Verbal Learning and Verbal Behavior, 1971, 10, 587-596.

Cutting, J. E., Rosner, B. S., \& Foard, C. F. Perceptual categories for musiclike sounds: Implications for theories of speech perception. Quarterly Journal of Experimental Psychology, 1976, 28, 361-378.

DIEHL, $R$. The effect of selective adaptation on the identification of speech sounds. Perception \& Psychophysics, 1975, 17, 48-52.

Diehl, R. L., Elman, J. L., \& McCusker, S. B. Contrast effects on stop consonant identification. Journal of Experimental Psychology: Human Perception and Performance, 1978, 4, 599-609.

Diehl, R. L., Lang, M., \& Parker, E. M. A further parallel between selective adaptation and contrast. Journal of Experimental Psychology: Human Perception and Performance, in press.

Eimas, P. D. Speech perception in early infancy. In L. B. Cohen \& P. Salapatek (Eds.), Infant perception. New York: Academic Press, 1975.

Eimas, P. D., \& Corbit, J. D. Selective adaptation of linguistic feature detectors. Cognitive Psychology, 1973, 4, 99-109.

Eimas, P. D., \& Miller, J. L. Effects of selective adaptation on the perception of speech and visual patterns: Evidence for feature detectors. In R. D. Walk \& H. L. Pick, Jr. (Eds.), Perception and experience. New York: Plenum, 1978.

Eimas, P. D., Siqueland, E. P., Jusczyk, P., \& Vigorito, J. Speech perception in infancy. Science, 1971, 171, 303-306.

Fowler, C. A., Rubin, P. E., Remez, R. E., \& Turvey, M. T. Implications for speech production of a general theory of action. In B. Butterworth (Ed.), Language production. New York: Academic Press, in press.

Fry, D. B., Abramson, A. S., Eimas, P. D., \& Liberman, A. M. The identification and discrimination of synthetic vowels. Language and Speech, 1962, 5, 171-189.

GanONG, W. F., III. The selective adaptation effects of burstcued stops. Perception \& Psychophysics, 1978, 24, 71-83.

Hall, L. L., \& Blumstein, S. E. The effect of syllabic stress and syllabic organization on the identification of speech sounds. Perception \& Psychophysics, 1978, 24, 137-144.

HAYES, W. L. Statistics for the social sciences. New York: Holt, Rinehart and Winston, 1973.

Hecaen, H., \& Albert, M. L. Human neuropsychology. New York: Wiley, 1978

KAY, R. H., \& MATthews, D. R. On the existence in human auditory pathways of channels selectively tuned to the modulation present in frequency-modulated tones. Journal of Physiology, 1972, 225, 657-677.

Liberman, A. M. Some characteristics of perception in the speech mode. In D. A. Hamburg (Ed.), Perception and its disorders: Research publications of the Association for Research in Nervous and Mental Disease (Vol. 48). Baltimore: Williams \& Wilkins, 1970.

Miller, G. A., \& Nicely, P. E. An analysis of perceptual confusions among some English consonants. Journal of the Acoustical Society of America, 1955, 27, 338-352.

OHMan, S. E. G. Coarticulation in VCV utterances: Spectrographic measurements. Journal of the Acoustical Society of America, $1966,39,151-168$.

Ohman, S. E. G. Numerical model of coarticulation. Journal of the Acoustical Society of America, 1967, 41, 310-320.

Perkell, J. S. Physiology of speech production. Cambridge: MIT Press, 1969.

Pisoni, D. B. Auditory short-term memory and vowel perception. Memory \& Cognition, 1975, 3, 7-18.

REMEz, R. E. Adaptation of the category boundary between speech and nonspeech: A case against feature detectors. Cognitive Psychology, 1979, 11, 38-57.

Rudnicky, A. I., \& Cole, R. A. Adaptation produced by connected speech. Journal of Experimental Psychology: Human Perception and Performance, 1977, 3, 51-61.

SAwUSCH, J. R. Selective adaptation effects on end-point stimuli in a speech series. Perception \& Psychophysics, 1976, 20, 61-65.

Sawusch, J. R. Peripheral and central processes in selective adaptation of place of articulation in stop consonants. Journal of the Acoustical Society of America, 1977, 62, 738-750.

Sawusch, J. R., \& Nusbaum, H. C. Contextual effects in vowel perception 1: Anchor-induced contrast effects. Perception \& Psychophysics, 1979, 25, 292-302.

Schmidt, F. O., Dev, P., \& Smith, B. H. Electrotonic processing of information by brain cells. Science, 1976, 193, 114-120.

Simon, H. J., \& Studdert-Kennedy, M. Selective anchoring and adaptation of phonetic and nonphonetic continua. Journal of the Acoustical Society of America, 1978, 64, 1338-1357.

Singh, S. Cross-language study of perceptual confusions of plosive phonemes in two conditions of distortion. Journal of the Acoustical Society of America, 1966, 40, 635-656.

Studdert-Kennedy, M., \& Shankweiler, D. P. Hemispheric specialization for speech perception. Journal of the Acoustical Society of America, 1970, 48, 570-594.

TARTter, V. C., \& Eimas, P. D. The role of auditory and phonetic feature detectors in the perception of speech. Perception \& Psychophysics, 1975, 18, 293-298.

VINEGRAD, M. D. A direct magnitude scaling method to investigate categorical versus continuous modes of speech perception. Language and Speech, 1972, 15, 114-121.

WiCkelgren, W. Distinctive features and errors in short-term memory for consonants. Journal of the Acoustical Society of America, 1966, 39, 388-398.

Woodworth, R. S. Experimental psychology. New York: Holt, 1938.

\section{NOTES}

1. The use of a rating procedure has shown that the effects of selective adaptation can be observed throughout an entire category, not only at the boundary stimuli (Sawusch, 1976).

2. See, also, a discussion by Schmidt, Dev, and Smith (1976) of the "quiet revolution" in the understanding of information processing by neurons. The widely influential assumption that perceptual primitives and individual neuron functions are correlated may be questionable on neurophysiological grounds.

(Received for publication June 12, 1979; revision accepted October $25,1979$. 